\title{
Porraz Gómez, Iván Francisco (2016). Más allá del sueño americano. Jóvenes migrantes retornados a Las Margaritas, Chiapas. México: IMJUVE, SEDESOL, CESMECA-UNICACH
}

\author{
Luis Rodríguez-Castillo*
}

6 T $\begin{aligned} & \text { endencias" es un poema de Antonio Bueno } \\ & \text { Tabía que forma parte del libro Signo sin } \\ & \text { lengua, publicado por la editorial Casus Belli }\end{aligned}$ (Bueno, 2016: 43-44), del cual tomo algunas estrofas para comentar/interpretar la investigación que mereció el premio en la categoría de doctorado en la séptima edición, año 2015, del Concurso Nacional de Tesis sobre Juventud. Cito:

\section{¿Qué línea seguir para llegar a ninguna parte sin estar en algún sitio?}

Quizás sea la pregunta más pertinente para tratar de definir a una población lábil que se inserta en complejos y contradictorios escenarios para tratar de cruzar o seguir una línea que llamamos frontera y divide a dos países. La población que se estudia en este libro no podría ser más diversa: jóvenes, algunos de los cuales ya vienen de un proceso de migración y retorno interno asociado al desplazamiento provocado por el movimiento zapatista de 1994, que llegaron siendo niños a la cabecera municipal de Las Margaritas, Chiapas, de una raigambre étnica y comunitaria, que crecieron en un lugar semirural — nos dice el autoro semiurbano, y que ahora migran, van y vienen hacia "ninguna parte"; esa que se habita en la imaginación del "sueño americano" y que el autor, siguiendo a Deleuze y Parnet, encuentra también como líneas de fuga.

"Qué líneas seguir" para situar un fenómeno que es una acuciante problemática social sobre la cual se escribe y describe mucho en sus procesos centrales, tales como: la situación juvenil, la situación migrante, la situación étnica, la situación marginal o la situación transnacional, sobre las cuales se llega, las más de las veces, a elegantes periodizaciones o categorizaciones que al final de cuentas resultan poco explicativas y son aún muy descriptivas. Destaca, por ejemplo, un artículo publicado en 2004 de Jorge Durand —uno de los grandes tatics del estudio del fenómeno migratorio-, en donde "analiza" la migración de retorno y construye una categorización como sigue: l) los que regresan de manera definitiva y voluntaria, 2) los trabajadores temporales, 3) el retorno transgeneracional, 4) los que retornan en condiciones forzadas, y 5) los fracasados (Durand 2004: 105-107).

Pues bien, la atención a esas situaciones y el intento de superar las limitaciones antes señaladas son las

* Luis Rodríguez Castillo. Centro de Investigaciones Multidisciplinarias sobre Chiapas y la Frontera Sur de la Universidad Nacional Autónoma de México.Correo electrónico: lurodri@unam.mx

Recibida: 7 de marzo de 2017 
tareas que se plantea Iván Porraz en el primer capítulo de la obra, luego de una breve introducción de la que destaco dos premisas fundamentales que guiaron el proceso de investigación: en primer lugar, que lo que ocurre en Chiapas —es producto de procesos de magnitud macro" y, en segundo lugar, que el "campo cultural es el privilegiado para el análisis de los jóvenes- (p. 23). Entonces - si acaso interpreto de manera correcta al autor-, en su primer capítulo, titulado "Juventud y migración. Una construcción teórica desde el conjuro situacional", a fin de evitar "llegar a ninguna parte", la propuesta se sitúa en el entrecruce interdisciplinario para analizar las consecuencias empíricas de ambas premisas: aquello que, viniendo del mundo global, trastoca el mundo cotidiano, familiar y comunitario de los jóvenes, así como sus prácticas y construcciones identitarias a partir del retorno migratorio o, puesto en otros términos, los significados que adquiere desde la diferencia —estilos adoptados en el lugar al que migraron- la reinserción y los rejuegos culturales de lo que implica ser joven en el espacio de origen y retorno, aspecto que, desde sus propias - líneas de fuga-, el autor denomina "múltiples retornos".

Continúo con otra estrofa de "Tendencias":

¿Qué línea seguir

para no dibujar una letra

y permanecer en silencio?

Si en la introducción y en el capítulo primero se denotan claramente las preocupaciones de corte antropológico, en el segundo capítulo, titulado "Las migraciones contemporáneas en la sociedad chiapaneca: causas y efectos", presenta el más puro estilo sociológico desde las teorías de la llamada "nueva economía de la migración”, mostrando el contexto y un diagnóstico de la creciente migración en la entidad. Datos, cifras y porcentajes se utilizan para no "permanecer en silencio" sobre las situaciones de vulnerabilidad que constituyen el contexto relevante de la juventud en la entidad y que "explican" el proceso migratorio: pobreza, desempleo, falta de tierras y la entrada de Chiapas, afirma siguiendo a Villafuerte, "por la puerta trasera de la globalización" (p. 81).

En esa misma tesitura, aunque ahora inclinándose a la historia social, el autor recupera la experiencia local de la migración en el capítulo tercero, "Del sur al norte: la migración en Las Margaritas, Chiapas". Con ello quiero decir que, además de los datos, cifras y porcentajes, el autor echa mano de sus fuentes primarias antropológicas para dar cuenta de los móviles y las formas que adquirió la migración, primero interestatal e intramunicipal y luego internacional, de los margaritenses. Al igual que en el capítulo anterior, buscando motivos antes que explicaciones, en este capítulo nos muestra algunos de los efectos: la transmutación de identidades, la experiencia de quienes pasan de ser migrantes retornados a polleros, la nueva dinámica "empresarial" y logística que implica el viaje y la decisión de "dar el brinco".

Para seguir hablando del libro cito otro fragmento de "Tendencias":

\section{¿Qué línea seguir para dejar de ser punto e inventar un sonido que signifique nada?}

Ésta parecer ser la pregunta que recubre la actividad de investigación en campo que se le presenta al colega Porraz y desembrolla en el capítulo cuarto, "Jóvenes migrantes: la irrupción de nuevas trayectorias socioculturales", que tiene como punto de partida la reflexión acerca de las capacidades para proyectar teoría sobre la realidad y extraer teoría de las particularidades espaciales y socioculturales de los jóvenes estudiados, que inventan algo nuevo que el autor denomina la "construcción cultural migratoria emergente" (p. 146). Si lo ha logrado o no es un asunto que dejo a la consideración de quienes lean este texto, pero deseo destacar algunas aristas que me parecen de interés.

La primera de ellas es que, más allá de la abigarrada referencia a autores en un mismo parágrafo, por ejemplo a Bauman, Sennett o Beck, lo que llama poderosamente 
la atención es que por momentos desmonta verdades consabidas sobre las causas de la migración expuestas en el segundo capítulo, aproximando su argumentación a las experiencias y deseos de los jóvenes, como "vivir situaciones nuevas a las habituales en la comunidad" (p. 158).

Otro aspecto a destacar es que en algunas investigaciones se presentan entramados de conceptos sobre los que nos preguntamos a qué realidad responden, mientras que en este libro el autor muestra su capacidad de extraer información relevante de dos fuentes empíricas; por una parte, las llamadas y los comentarios de la radio local XEVFS AM La Voz de la Frontera Sur, para ofrecernos un panorama de la migración desde Las Margaritas a Estados Unidos $y$, por el otro, la trayectoria vivencial de los jóvenes margaritenses con experiencia migratoria.

En tercer lugar, con estos materiales el autor nos deja escuchar los sonidos preñados de significados de los migrantes jóvenes margaritenses. En ellos, sin hacer apología de las penurias, aborda aspectos significativos de la vida "allá" de los jóvenes migrantes: el trabajo, las actividades en el tiempo libre y las relaciones de pareja, así como los aspectos que "acá" preocupan a los que se quedan: el consumo de drogas y los nuevos materiales culturales que se adquieren y se ven como peligrosos en cuanto trastocan normas y valores locales.

¿Qué línea seguir?
¿la del árbol al desnudarse
en el otoño de un libro
o de repente ser otra cosa
aliento que inesperado ocurre
de algo no visto que pasa
y termina
para comenzar?

"Que pasa / y termina / para comenzar", así es la realidad presentada en el quinto capítulo "iMi vida ya no es la misma, es mejor, no lo sé! Jóvenes migrantes retornados; familia y entorno social", en el que el autor expone la trama de las tensiones que se hacen evidentes, así como "los significados que tiene el retorno en sus vidas presentes y sus posibles horizontes" (p. 181). Este tema adquiere centralidad en el marco de procesos de criminalización de migrantes y retornados, así como de la puesta en marcha de programas de securitización de los Estados a fin de frenar la migración. Entre los cambios en la biografía, en lugar de ser considerados "aliento que inesperado ocurre", el autor observa la precariedad temporal de la experiencia y que aquellos “aceptan volver a lo mismo, transitan en líneas impredecibles" (p. 186).

¿Por qué se retorna? Tiene múltiples respuestas; desde las malas experiencias con la migración y los coyotes, hasta los secuestros en "los Estados", los sentires y extrañamientos no sólo culturales, sino principalmente a los que se quedan "acá", o el orgullo y heroísmo que se retrata en los relatos del retorno: ir, regresar con vida y con algunos ahorros.

Pero también aborda en este acápite las tensiones que se observan ante la "nueva forma de ser" joven: tatuajes, vestimenta diferente, otras ideas, en fin, nuevas corporalidades que generan tensiones a partir de las percepciones y asociaciones en los imaginarios locales de los retornados como delincuentes y por su contraposición a la "tradición" comunitaria, ambos temas definidos desde una perspectiva adultocéntrica que genera para los jóvenes esos sentimientos de extrañamiento e incomodidad de estar de regreso. Antes que quedarse en una contraposición entre modernidad/ tradición, o en el laberinto de la situación estructural que explica la falta de oportunidades laborales para los retornados, realiza una visionaria reflexión en torno a cómo esas situaciones muestran la vulnerabilidad de la situación juvenil en Las Margaritas, en donde, siguiendo a Butler, afirma que se muestran como "población superflua" para el Estado mexicano y como "vidas precarias"; son los nuevos enemigos para el Estado norteamericano.

Entonces, la siguiente estrofa del multicitado poema,

$$
\begin{gathered}
\text { ¿Qué línea seguir } \\
\text { hasta no seguir línea ninguna? } \\
\text { ¿Qué línea seguir... } \\
\text { Qué línea? }
\end{gathered}
$$


Me sirve para comentar el capítulo sexto, "Pensar la migración de retorno, pensar a los jóvenes migrantes del sur: algunas conclusiones para debatir", en donde el autor muestra un irredento espíritu, que a cualquier investigador - joven, maduro o viejo - bien nos valdría guardar. Reivindica su responsabilidad de dar a conocer una realidad social particular, pero también de mantener un espíritu crítico y ético, al señalar que pensar en la migración significa "pensar que sus actores protagónicos [están] reducidos a una 'nuda vida"' (p. 219).

Y para dejar "puntos suspensivos", como dice el Dr. Porraz, o siguiendo el poema "Tendencias", "seguir una línea / hasta no seguir ninguna” nos invita a reflexionar sobre las posibilidades de una agenda de investigación que él delinea en cinco aspectos, que por su relevancia no mencionaré para despertar la curiosidad por la lectura de Más allá del sueño americano. Jóvenes migrantes retornados a Las Margaritas, Chiapas.

\section{Referencias}

Bueno Tabía, Antonio (2016). Signo sin lengua. México: Casus Belli.

Durand, Jorge (2004). "Ensayo teórico sobre la migración de retorno. El principio del rendimiento decreciente". En Cuadernos Geográficos, 35(2); 103-116. 